REM-Schlaf-Verhaltensstörungen \section{Gefährliche Träume}

Sie schlagen um sich, strampeln, schreien - und schlafen trotzdem felsenfest. Patienten mit REM-Schlaf-Verhaltensstörungen leben buchstäblich ihre Träume. Dabei ist nicht nur das Verletzungsrisiko hoch, viele entwickeln innerhalb weniger Jahre Parkinson oder eine Demenz.

E beginnt oft im Alter von 55 bis 60 Jahren und ist vor allem für die Bettgenossen gefährlich: Wenn die Motorik im REM-Schlaf nicht mehr abgeschaltet ist, dann fliegen Fäuste, es gibt Tritte, und manchmal wird sogar gebrüllt. Die Patienten wissen von all dem nach dem Aufwachen oft nichts mehr, sie erinnern sich allenfalls noch an einen lebhaften Traum. Die akute Verletzungsgefahr bei den Betroffenen und ihren Lebenspartnern ist jedoch nur das eine Problem, so Professor Joan Santamaria, Barcelona. Denn bei vielen, vermutlich sogar bei den meisten der Patienten kündigt die REM-SchafVerhaltensstörung (RBD, rapid-eye-movement sleep behaviour disorder) den Beginn einer neurodegenerativen Erkrankung an, und zwar fast ausschließlich aus dem Parkinson-Spektrum. Das wird aus einer Reihe von Arbeiten deutlich. So hatte in einer retrospektiven Studie etwa die Hälfte der Patienten, bei denen in einem Schlafzentrum eine RBD diagnostiziert worden war, innerhalb von zwölf Jahren nach dem Beginn der Symptome eine Synucleinopathie wie Parkinson, Multisystematrophie (MSA) oder LewyKörperchen-Demenz entwickelt.

Eine andere Analyse bei 54 Patienten mit RBD ergab, dass mehr als die Hälfte (61\%) der neurologisch noch unauffälligen Patienten Riechstörungen hatten, wie sie auch in der präklinischen Phase bei Parkinson-Kranken typisch sind. RBD-Patienten ohne Riechstörungen konvertierten dabei seltener in eine neurodegenerative Erkrankung als solche mit Hyposmie. Hinweise auf einen Zusammenhang der Schlafstörung mit Parkinson liefert zudem die Bildgebung. So verringerte sich die Konzentration von Dopamintransporter-Proteinen in nigrostriatalen Bereichen bei RBD-Patienten innerhalb von drei Jahren um etwa $20 \%$, bei gleichaltrigen Kontrollpersonen ohne RBD nur um $8 \%$. Auch hier zeigte sich: Vor allem solche Patienten mit einem starken Rückgang der Werte entwickelten Parkinson. Dies heißt jedoch nicht, dass sich die anderen Patienten sicher fühlen dürfen: Nicht selten mündet die RBD auch in einer Lewy-Körperchen-Demenz. Santamaria zeigte Daten einer Studie von 231 Patienten mit RBD. Bei allen Patienten, die zusätzlich eine Lewy-KörperchenDemenz hatten, war die RBD schon vor der Demenz aufgetreten. Dagegen war nur bei jedem fünften Parkinson-Kranken mit RBD die Schlafstörung vor der ParkinsonDiagnose aufgefallen, bei den meisten trat sie also erst hinterher auf.

Unterschiede gibt es auch bei der Prävalenz der RBD bei den verschiedenen neurodegenerativen Erkrankungen: Je nach Studie und Erkrankungsdauer der Patienten liegt diese bei 15-60\% für Parkinson-Kranke. Dagegen zeigen praktisch alle MSA-Patienten sowie mehr als $70 \%$ der Patienten mit Lewy-KörperchenDemenz REM-Schlaf-Verhaltensstörungen. Bei anderen Demenzformen wie der Alzheimer-Erkrankung kommt die RBD dagegen nur selten vor. Was kann man aus all diesen Daten schließen?

\section{Die RDB hilft bei Vorhersage neurodegenerativer Erkrankungen}

Zunächst deutet eine RBD bei älteren Menschen auf den Beginn einer Synucleinopathie hin, wobei die RBD allein noch keinen Aufschluss darüber gibt, welche Patienten tatsächlich erkranken und wenn ja, ob sie Parkinson, MSA oder eine LewyKörperchen-Demenz bekommen. Zusätzliche Hinweise, so Santamaria, können dann aber in die richtige Richtung führen und eine Frühdiagnose erleichtern. Haben RBD-Patienten zum Beispiel schon leichte kognitive Probleme, spricht sehr vieles für den Beginn einer Lewy-KörperchenDemenz. Dagegen scheint eine Hyperechogenität im transkraniellen Ultraschall bei RBD eher für eine Parkinson-Erkrankung zu stehen. Schließlich, so der Neurologe, kann die RBD bei der Diagnose

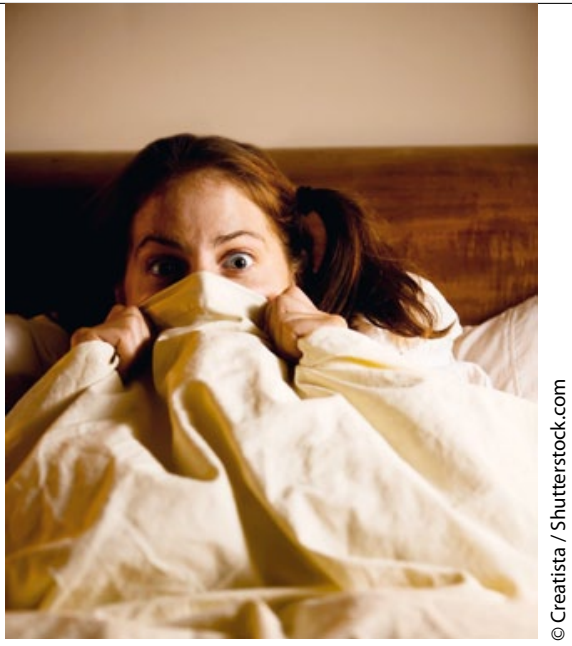

Für Bettgenossen von Menschen mit REM-Schlaf-Verhaltensstörungen kann die Nacht zum Alptraum werden.

einer Synucleinopathie helfen. Haben etwa Patienten mit Verdacht auf Parkinson auch eine RBD, so kann man davon ausgehen, dass der Verdacht richtig ist. Die RBD hilft entsprechend auch, eine Lewy-Körperchen-Demenz von einer Alzheimer-Demenz zu unterscheiden.

Dennoch bleibt die Frage, weshalb die REM-Schlafstörung bei manchen Patienten lange vor den Symptomen einer neurodegenerativen Erkrankung auftritt, bei anderen erst viel später oder gar nicht. Santamaria vermutet, dass dies möglicherweise an den unterschiedlichen Verlaufsformen der einzelnen Erkrankungen liegt. So ist die RBD bei Parkinson praktisch nur beim rigid-akinetischen Typ ein Frühsymptom, während sie sich beim tremordominanten Typ fast immer nach den motorischen Symptomen manifestiert. Dies könnte wiederum daran liegen, dass sich die parkinson-typischen AlphaSynuclein-Ablagerungen in unterschiedlicher Weise vom Hirnstamm ausgehend im Gehirn ausbreiten und dabei zu unterschiedlichen Zeitpunkten Regionen lahmlegen, die für die Unterdrückung der Motorik im REM-Schlaf nötig sind.

Trotzdem bleiben noch viele Zusammenhänge unklar. So ist die RBD offenbar kein dopaminerges Phänomen, die Störungen werden weder von ParkinsonMedikamenten noch von der subthalamischen tiefen Hirnstimulation gelindert. Man darf hier also auf die weitere Forschung gespannt sein. (Siehe auch Interview auf Seite 18.) Thomas Müller

Symposium „Neurology and sleep-wake disorders" 22. ENS-Kongress, Prag, 12.6.2012 